\title{
SINOPSIS DE ESPECIES MEXICANAS DEL GÉNERO XYLEBORUS EICHHOFF, 1864 (COLEOPTERA: CURCULIONIDAE: SCOLYTINAE)
}

\section{Mauricio PÉREZ SilVA, ${ }^{1}$ Armando EQUihUA MARTÍNEZ, ${ }^{1}$ Edith G. ESTRAdA VENEGAS, ${ }^{1}$ ANa LiLIA MUÑOZ VIVEROS, ${ }^{2}$ JoRge M. VALDEZ CARRAZCO, ${ }^{1}{ }^{3}$ ULIO SÁNCHEZ ESCUDERO ${ }^{1}$ y THOMAS H. ATKINSON $^{3}$}

\author{
${ }^{1}$ Instituto de Fitosanidad, Colegio de Postgraduados, Campus Montecillo. Km. 36.5 Carretera México-Texcoco, \\ 56230, Montecillo, Texcoco, Estado de México, México <mau_2986@hotmail.com>; <equihuaa@colpos.mx>; \\ <edith_ev@yahoo.com.mx>; <jvaldez@colpos.mx>; <clarijul@hotmail.com> \\ ${ }^{2}$ Laboratorio de Control de Plagas, FES- Iztacala-UNAM. Av. De los Barrios No. 1, 54090, Los Reyes Iztacala, \\ Tlalnepantla, Estado de México, México <analiliamunoz@hotmail.com> \\ ${ }^{3}$ University of Texas Insect Collection, 3002 Lake Austin Blvd., Austin, TX 78703, U.S.A. \\ $<$ thatkinson.austin@gmail.com> \\ Recibido: 19/10/2014; aceptado: 22/01/2015
}

Pérez Silva, M., Equihua Martínez, A., Estrada Venegas, E. G., Muñoz Viveros, A. L., Valdez Carrazco, J. M., Sánchez Escudero, J. \& Atkinson, T. H. 2015. Sinopsis de especies mexicanas del género Xyleborus Eichhoff, 1864 (Coleoptera: Curculionidae: Scolytinae). Acta Zoológica Mexicana (n. s.), 31(2): 239-250.

RESUMEN. Dentro de Scolytinae, uno de los grupos de mayor importancia en áreas tropicales es el género Xyleborus, reconocidos por sus hábitos alimentarios como coleópteros ambrosiales. En México pocos estudios se han enfocado a dicho género; por lo anterior el presente trabajo tuvo como objetivo realizar un listado taxonómico de las especies de Xyleborus presentes en México, así como su distribución actual y los hospederos de las especies reportadas. Para llevar a cabo dicho estudio, se consultaron colecciones científicas, se realizaron recolectas en algunas localidades de 6 estados de la República Mexicana campo y se revisó la literatura sobre Scolytinae más actualizada. Los organismos se identificaron y corroboraron, según el caso, con claves taxonómicas especializadas. Se encontraron 18 especies del género Xyleborus, de las cuales $X$. bispinatus, y $X$. vismiae se reportan por primera vez en México; $X$. declivis, $X$. horridus, $X$. intrusus, $X$. macer y $X$. posticus se registraron por primera vez en algunos estados de la República Mexicana. El estado en donde se reporta mayor número de especies es Veracruz con 14. La distribución de las especies mexicanas de Xyleborus se complementa con mapas, además de incluir una lista de hospederos, de acuerdo con el material entomológico y la literatura consultada.

Palabras clave: Coleópteros ambrosiales, áreas tropicales, listado taxonómico.

\section{INTRODUCCIÓN}

La subfamilia Scolytinae se reconoce por poseer especies consideradas plagas de importancia económica. Se estima que en las áreas forestales de México, 90 \% de los daños provocados por plagas, son causados por descortezadores (Burgos-Solorio \& Equihua 2007). Sin embargo, de las aproximadamente 870 especies de Scolytinae reportadas para el país (Atkinson 2012), sólo algunas, como las pertenecientes a los géneros Dendroctonus, Ips, Phloeosinus,
Pérez Silva, M., Equihua Martínez, A., Estrada Venegas, E. G., Muñoz Viveros, A. L., Valdez Carrazco, J. M., Sánchez Escudero, J. \& Atkinson, T. H. 2015. Synopsis of Mexican species of the genus Xyleborus Eichhoff, 1864 (Coleoptera: Curculionidae: Scolytinae). Acta Zoológica Mexicana (n. s.), 31(2): 239-250.

ABSTRACT. Within Scolytinae, one of the most important groups in tropical areas is the genus Xyleborus, recognized for their feeding habits as ambrosial beetles. In Mexico, only a few studies have been focused on the genus. Therefore the present work had the purpose a taxonomic list of species present in Mexico Xyleborus and its current distribution and hosts of the species reported. To carry out this study, scientific collections were revised; collections were performed in some localities 6 states of Mexico field and current literature on the genus revised. Species were identified with specialized taxonomic keys. In Mexico there are 18 species of Xyleborus, of which $X$. bispinatus and $X$. vismiae are reported for the first time in the country; $X$. declivis, $X$. intrusus, $X$. horridus, $X$. macer and $X$. posticus were recorded for the first time in some Mexican states. The state where most species of the genus are reported is Veracruz with 14. The distribution of the Mexican species of Xyleborus is complemented by maps, and includes a list of hosts, according to the entomological material the literature. Key words: Ambrosial beetles, tropical areas, taxonomic list.

Scolytus y Pityophthorus, se consideran de importancia económica en coníferas (Cibrián et al. 1995). Otros géneros, como Xyleborus, incluyen especies de igual importancia en los trópicos. No obstante, la función principal de los escolitinos es la de participar en los procesos de descomposición de la materia orgánica, atacando árboles muertos, moribundos, débiles o trocería recién cortada (Equihua \& Burgos 2002). De acuerdo con sus hábitos alimenticios, los escolitinos pueden ser xilomicetófagos, fleófagos, espermatófagos y mielófagos (Atkinson \& 
Equihua 1986a y b). Los xilomicetófagos también son conocidos como coleópteros ambrosiales, debido a que llevan consigo hongos que se cultivan en el interior de las galerías y les sirven de alimento a ellos mismos (Rangel et al. 2012). La subtribu Xyleborina comprende una gran diversidad de especies de hábitos ambrosiales, de las cuales las pertenecientes al género Xyleborus son de las más importantes y complejas, aunque en muchos casos estas especies son secundarias, en algunos otros, pueden tornarse como plagas de gran impacto (Lombardero 1996; Wood 1982).

El género Xyleborus fue descrito en 1864, por Eichho$\mathrm{ff}$, dentro del cual se incluyeron algunas especies originalmente descritas en el género Bostrichus. Con el paso de los años, se han descrito muchas especies del género y han ocurrido cambios taxonómicos, apoyados principalmente en caracteres morfológicos (Bright 1968). Wood (1982) propuso que el género Xyleborus se dividiera en cuatro subgéneros: Ambrosiodmus, Euwallacea, Neoxyleborus y Coptoborus, de los cuales todos, excepto Neoxyleborus, fueron tomados en cuenta como géneros distintos (Wood 1986). Hulcr et al. (2007) plantearon la resurrección del género Anisandrus, el cual se consideraba como sinónimo de Xyleborus. Actualmente, se reportan aproximadamente 534 especies del género a nivel mundial, aunque los cambios taxonómicos, como los mencionados anteriormente, hacen que el número de especies varíe constantemente (Hulcr et al. 2007).

En México, el género Xyleborus se ha estudiado poco; sin embargo, los trabajos de Wood (1982), Romero et al. (1997) y Equihua \& Burgos (2002) abarcan aspectos fundamentales sobre escolitinos (taxonomía, biología y ecología), incluyendo información sobre Xyleborus a nivel nacional. Otros estudios enfocados en regiones particulares de México o en tipos de vegetación específicos (Atkinson et al. 1986a, Atkinson et al. 1986b, Atkinson \& Equihua 1986a, Atkinson \& Equihua 1986b, Estrada \& Atkinson 1988, Noguera-Martínez \& Atkinson 1990, Burgos-Solorio \& Equihua 2007 y Pérez-De La Cruz et al. 2009a) también señalan a dicho grupo. En años recientes ha aumentado la información sobre el género en México, debido a que ciertas especies representan un riesgo para algunas plantaciones forestales de importancia económica, tal es el caso de Xyleborus affinis, $X$. ferrugineus y $X$. volvulus, los cuales pueden afectar árboles de Cedrela odorata y plantaciones de cacao (Pérez-De La Cruz et al. 2009a; Rangel et al. 2012); X. glabratus aunque no se encuentra reportada en México, de acuerdo con la Dirección General de Sanidad Vegetal (2014), se considera como un riesgo potencial para las plantaciones de aguacate en el país, ya que se encuentra en calidad de plaga cuarentenaria.
Debido al creciente interés e importancia del género Xyleborus en México y a que la información sobre dicho grupo es escasa, en el presente estudio se da a conocer un listado de las especies del género Xyleborus presente en México, así como su distribución actual y los hospederos de las especies reportadas.

\section{MATERIAL Y MÉTODOS}

Para realizar el presente estudio se utilizó información obtenida de tres medios: 1) colecciones científicas nacionales, 2) recolecta de campo y 3) revisión bibliográfica. En forma complementaria, se solicitaron ejemplares de especies no representadas en las colecciones mexicanas.

Se consultaron las siguientes colecciones: colección entomológica de Fitosanidad del Colegio de Postgraduados (CIIFIT), la colección de coleópteros y la de insectos forestales de la Escuela Nacional de Ciencias Biológicas, del Instituto Politécnico Nacional (ENCB-IPN), y el material de escolitinos de la Colección Nacional de Insectos (CNIN) del Instituto de Biología, de la Universidad Nacional Autónoma de México. De cada colección se obtuvo el número de especies y los datos de colecta, que incluyen localidad y hospederos.

La recolecta de insectos se realizó durante el segundo semestre de 2013, en algunas localidades de seis estados de la República Mexicana, las cuales fueron: la Reserva Ecológica de "La Chontalpa" y el Colegio de Postgraduados, Campus Tabasco, en Tabasco; Parque Nacional “El Tepozteco”, municipio de Tepoztlán, en Morelos; Estación de Biología Tropical "Los Tuxtlas", San Andrés Tuxtla, en Veracruz; dos localidades en la Selva Lacandona, Metzabock y Nahá, en Chiapas; Ocote de Cadena, Petatlán, en Guerrero; y Gómez Farías, en Tamaulipas.

En cada uno de los sitios se colocaron trampas tipo embudo con luz negra como atrayente y un recipiente recolector en la base con alcohol a 70\% a $1.5 \mathrm{~m}$ de altura. La lámpara se encendió alrededor de las 18:00 h y se recogió a la mañana siguiente, no obstante, la trampa se dejó en el sitio para que durante el día el alcohol pudiera actuar como atrayente. Este procedimiento se llevó a cabo durante 2 o 3 días, en cada localidad. En algunos casos se extrajeron los insectos de trozas recién cortadas. Todo el material se conservó en frascos con alcohol a 70\% para transportarse e identificarse. Los ejemplares se depositaron en la Colección Entomológica del Colegio de Postgraduados Campus Montecillo.

Se consultaron las publicaciones más recientes sobre escolitinos de México (Atkinson, 2012, Atkinson et al. 1986a, Atkinson et al. 1986b, Atkinson \& Equihua 1986a, Atkinson \& Equihua 1986b, Burgos-Solorio \& Equihua 2007, Equihua \& Burgos 2002, Estrada \& Atkinson 1988, 
Noguera-Martínez \& Atkinson 1990, Pérez-De La Cruz et al. 2009a, Romero et al. 1997 y Wood 1982). La información se complementó con la contenida en la página web Bark and Ambrosia Beetles (http://www.barkbeetles. info/about.php) de T. H. Atkinson (University of Texas).

Todos los ejemplares recolectados se identificaron taxonómicamente y los que se revisaron en las colecciones científicas fueron corroborados, analizando la morfología externa, con el apoyo de claves especializadas (Wood 1982, Rabaglia 2006 y Pérez-De La Cruz et al. 2009a); para lo cual se tomaron en cuenta: la coloración, el tamaño, el declive elitral, la ornamentación, la elevación pronotal, la dentición del margen anterior del pronoto y las estrías e interestrías de los élitros y el declive elitral.

De forma complementaria se anexa información sobre la distribución, los hospederos y la colección en donde se encuentran depositadas cada una de las especies mexicanas de Xyleborus (Apéndice 1); además se realizaron mapas de distribución con base en la información obtenida durante el presente estudio y la disponible página web del T. H. Atkinson, quien como coautor generó dichos mapas y son parte de la información que él ha considerado útil para una visión más integral de la distribución de las especies mexicanas, además incluyen información de Norte y Centroamérica, pero solo se contempla la referente a México (Apéndice 2).

\section{RESULTADOS}

Se reportan 18 especies del género Xyleborus Eichhoff para México, de las cuales dos se registran por primera vez en el país: Xyleborus bispinatus Eichhoff y X. vismiae Wood (Cuadro 1).

En la CIIFIT se encontraron 16 especies de Xyleborus, siendo la colección con mayor número de especies registradas, debido a que muchos de los ejemplares recolectados en los trabajos de escolitinos previos se deposi- taron en esta colección. De estas especies sólo Xyleborus bispinatus es nuevo registro para México. En la CNIN se encontraron seis especies de las cuales, $X$. bispinatus se reportan por primera vez en el país. Aunque esta especie se recolectó en la década de 1980, fue determina hasta el 2007 por T. H. Atkinson, razón por la cual en los trabajos anteriores no se había contemplado. En las colecciones de la ENCB-IPN se encontraron depositadas cuatro especies, $X$. affinis, $X$. ferrugineus, $X$. volvulus y $X$. scopulorum.

En campo se recolectaron 54 especímenes de $X$. affinis, 20 de $X$. ferrugineus, 1 de $X$. intrusus, 1 de $X$. posticus y 24 de $X$. volvulus.

En el estado de Veracruz se registraron 14 especies, considerado como el mejor representado, seguido por Chiapas, Campeche, Oaxaca, Tabasco y Tamaulipas, con 11, 9, 9, 8 y 7 especies, respectivamente (Fig. 1). En el presente estudio, por primera vez se reporta $X$. horridus en los estados de Chiapas, Guerrero y San Luis Potosí; $X$. intrusus en Baja California Norte y Baja California Sur; $X$. spinulosus en Tamaulipas; $X$. posticus en Morelos y $X$. declivis en Chiapas y Tabasco, aunque no se tiene registro de colecta ni publicación para este último (Atkinson 2014).

En México $X$. volvulus y $X$. ferrugineus se encuentran reportadas para 21 estados, seguidas por $X$. affinis y $X$. intrusus con registros para 19 y 14 especies respectivamente. A diferencia de las especies antes mencionadas, $X$. morulus, $X$. spathipennis, $X$. titubanter y $X$. vismiae sólo se restringen a uno o dos estados, además de contar con escasos registros (Fig. 2).

\section{DISCUSIÓN}

Wood (1982) reportó 22 especies del género Xyleborus en México; sin embargo, el número se redujo drásticamente, ya que en 1986 Wood separó a Ambrosiodmus Hopkins, Coptoborus Hopkins, Taurodemus Wood y Euwallacea

Cuadro 1. Especies del género Xyleborus registradas en México en el presente estudio.

Especies mexicanas del género Xyleborus Eichhoff

\begin{tabular}{ll}
\hline 1. Xyleborus affinis Eichhoff 1868 & 10. Xyleborus morulus Blandford 1898 \\
2. Xyleborus bispinatus Eichhoff $1868^{\S}$ & 11. Xyleborus palatus Wood 1974 \\
3. Xyleborus declivis Eichhoff 1869 & 12. Xyleborus posticus Eichhoff 1869 \\
4. Xyleborus discretus Eggers 1933 & 13. Xyleborus spathipennis Eichhoff 1868 \\
5. Xyleborus ferrugineus (Fabricius) 1801 & 14. Xyleborus spinulosus Blandford 1898 \\
6. Xyleborus horridus Eichhoff 1869 & 15. Xyleborus squamulatus Eichhoff 1869 \\
7. Xyleborus imbellis Blandford 1898 & 16. Xyleborus titubanter Schedl 1948 \\
8. Xyleborus intrusus Blandford 1898 & 17. Xyleborus vismiae Wood $1974^{\S}$ \\
9. Xyleborus macer Blandford 1898 & 18. Xyleborus volvulus (Fabricius) 1775 \\
\hline
\end{tabular}

$\S$ Especies registradas por primera vez para México. 


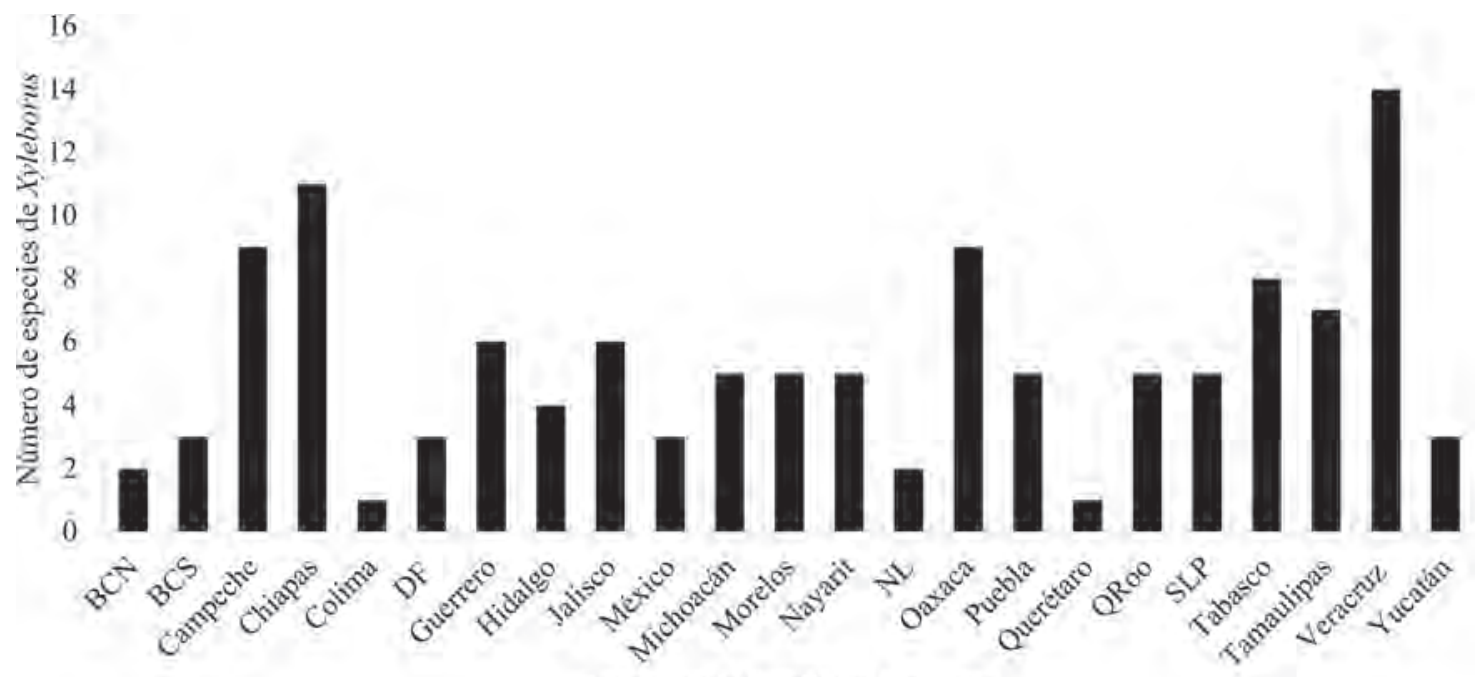

Estados de la República Mexicana

Figura 1. Número de especies de Xyleborus registradas en los diferentes estados de la República Mexicana; BCN: Baja California Norte, BCS: Baja California Sur, DF: Distrito Federal, NL: Nuevo León, QRoo: Quintana Roo y SLP: San Luis Potosí.

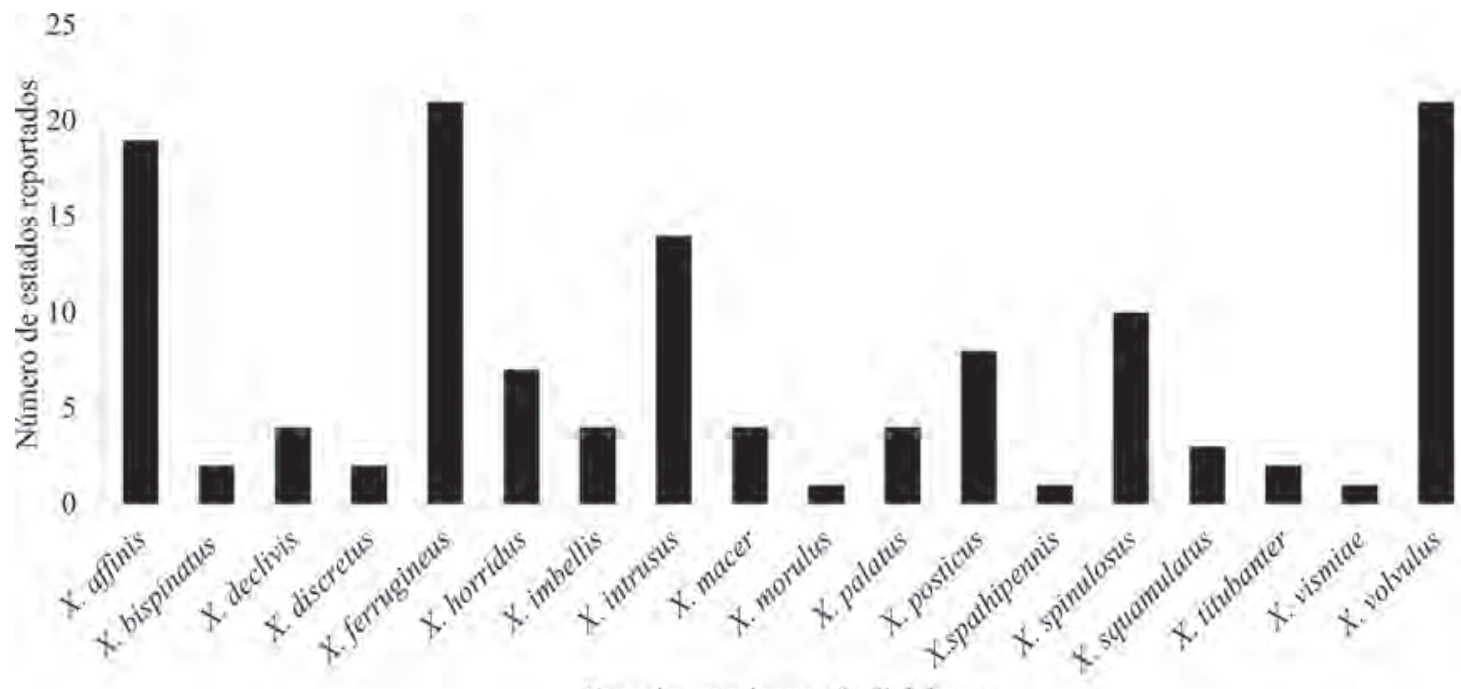

Especies mexicanas de Xyleborus

Figura 2. Número de estados correspondientes a los reportados en las especies mexicanas de Xyleborus.

Hopkins a partir de Xyleborus; a grandes rasgos, la separación se hizo con base en caracteres morfológicos, como las crenulaciones del pronoto, la forma y ornamentaciones del margen anterior del pronoto, dientes en la protibia y declive elitral (Wood 1986; Rabaglia et al. 2006). Por lo anterior, especies como Ambrosiodmus obliquus, A. rusticus, A. guatemalensis, Coptoborus pseudotenuis, $C$. vespatorius, $C$. tolimanus y Taurodemus sharpi ya no son consideradas dentro del género Xyleborus. Posteriormente Romero et al. (1997) hacen referencia a la presencia de 18 especies del género, sin embargo aún consideran a T. sharpi como parte de Xyleborus. Equihua \& Burgos (2002), en una actualización de Scolytinae en México, re- portan 17 especies de Xyleborus para México, en el cual ya no consideran a $T$. sharpi, contemplada por Romero et al. (1997), no obstante incluyen a Coptoborus pseudotenuis y no contemplan a Xyleborus morulus.

La clasificación de Wood (1986) había sido respetada, sin embargo Hulcr et al. (2007) dieron a conocer la resurrección de cuatro géneros de la subtribu Xyleborina, entre los cuales destaca Anisandrus Ferrari, ya que anteriormente era considerado como sinonimia del género Xyleborus. La separación de Anisandrus fue con base en el primer segmento del mazo antenal forma una costa circular en la cara anterior, los segmentos 2 y 3 no son visibles en la cara posterior del mazo antenal, la presencia de 
“copete" micangial pronotal, la forma lateral y dorsal del pronoto, y la forma terminal de los élitros. Con base en esta información, en el presente estudio ya no se considera a A. dispar como perteneciente al género Xyleborus. Estudios recientes han proporcionado información con base en la cual actualmente se consideran 34 géneros dentro de la subtribu Xyleborina (Hulcr \& Cognato 2010; Bright 2014), por lo que algunas especies del género Xyleborus se han removido a otros géneros. Esta nueva clasificación no ha afectado la diversidad de las especies reportadas en México.

Recientemente Atkinson et al. (2013), con base en la espina de la tercer interestría y la elevación pronotal, diferenciaron a $X$. bispinatus de $X$. ferrugineus, lo cual permitió separar ambas especies de la CIIFIT, ya que se comprobó que se encontraban mezcladas.

Dentro de la colección de coleópteros de la ENCB se encontraron dos ejemplares identificados como $X$. scopulorum, recolectados en 1946 en Puebla e identificados en 1969 por Bright D. E., pero esta especie se considera sinónimo de $X$. intrusus (Wood 1982, Rabaglia et al. 2006).

En el presente estudio se observó que dentro de $X$. volvulus aparentemente existen dos morfotipos, el primero de coloración café oscura a casi negra; las estrías discales son impresas, estría 1 en una fila semidefinida, las puntuaciones son pequeñas y profundas, y el declive es más empinado. En el caso del segundo morfotipo se observa una coloración café rojiza a café clara, las estrías son bien definidas y ligeramente impresas, puntuaciones más gruesas y declive empinado, pero menos que en el caso anterior. Wood (1982) menciona que originalmente existieron dos formas geográficas distintas, una distribuida en Norteamérica que coincide con el morfotipo 1 y la otra limitada a Centro y Sudamérica, la cual coincide con el morfotipo 2; además menciona que actualmente pueden estar mezclados. Debido a esto es indispensable hacer una caracterización detallada de estas poblaciones para definir su posición taxonómica definitiva.

La distribución del género Xyleborus se extiende por toda la República Mexicana (Apéndice 1), sin embargo, $X$. affinis, $X$. ferrugineus y $X$. volvulus presentan un rango bastante amplio, dado que se encuentran reportadas para casi todos los estados de México, incluso Wood (1982) menciona que las primeras dos se encuentran en todos los estados de México, aunque no se encontraron registros de su presencia en algunos de ellos. El rango tan amplio en la distribución de estas tres especies, se debe a que poseen la capacidad de infestar varias especies vegetales (Vázquez et al. 2003; Pulgarín 2012). A diferencia de otros escolitinos, como los fleófagos, los coleópteros ambrosiales no requieren un alto grado de especificidad en sus hospedantes, debido a que no son la fuente directa de su alimentación, sino los hongos que llevan consigo y que se cultivan dentro de las galerías, ya que estos se desarrollan en el duramen, constituido básicamente por células muertas. Por esta razón, el género Xyleborus tiene la ventaja de arribar a una gran diversidad de especies; asimismo, la especificidad es reducida (Atkinson \& Equihua 1986a).

En comparación con otros grupos de escolitinos en México, el género Xyleborus es representado de manera importante, en comparación con otros grupos de mayor interés económico, como Dendroctonus, en donde el número de especies reportadas para México es de 12 (Salinas-Moreno et al. 2010), mientras que para los géneros Scolytus e Ips se reportan 16 y 9 especies respectivamente; no obstante, hay géneros, como Pseudothysanoes o Pityophthorus, representados por más de 50 especies (Atkinson 2014).

Afinidades biogeográficas. Actualmente no se tiene conocimiento de los patrones biogeográficos del género Xyleborus en México, por lo que en el presente estudio, se hizo un primer acercamiento a la biogeografía del género en México; sin embargo, es conveniente revisar con mayor detenimiento la distribución y las condiciones que determinan la presencia de cada una de las especies en ciertas regiones, con un mayor número de recolectas, ya que el presente estudio se apoyó en gran medida en los registros previos.

Diversos autores hacen referencia a que el género Xyleborus se distribuye preferentemente en los ecosistemas tropicales y subtropicales (Wood 1982; Atkinson \& Equihua-Martínez 1986b), lo cual coincide con lo observado en el presente estudio, ya que la mayoría de las especies se encuentran distribuidas en áreas tropicales; con excepción de $X$. intrusus (Apéndice 2), la cual únicamente se ha reportado en ecosistemas con clima templado, asociada a los bosques de pino (Atkinson et al. 1986b). Se observó que 10 especies se distribuyen exclusivamente sobre la vertiente del Golfo de México, área que de acuerdo con Atkinson y Equihua (1986b) es el límite norte de la región Neotropical, hasta el sur de Tamaulipas; de estas especies $X$. declivis, $X$. discretus, $X$. macer, $X$. spathipennis, $X$. squamulatus, $X$. titubanter y $X$. vismiae (Apéndice 1 y 2) se reportan del centro de Veracruz hacia el sureste mexicano, área donde predomina clima cálido y húmedo (Rzedowski 1978); tanto X. posticus como X. horridus presentan una distribución más amplia, que se extiende por toda la vertiente del Golfo de México; mientras que $X$. imbellis, ha sido registrada del centro de Veracruz al sur de Tamaulipas, aunque Equihua y Burgos (2002) la reportan en Campeche y Quintana Roo no se encontraron registros específicos de su presencia (Apéndice 2), por lo que es indispensable definir su preferencia, ya que probablemente se pueda encontrar en regiones más templadas, 
debido a que fue reportada por Noguera-Martínez y Atkinson (1990) en bosque mesófilo de montaña y no por Atkinson y Equihua (1986b) en su estudio en un bosque tropical lluvioso, ambos casos en Veracruz. Aunque $X$. bispinatus se registra para la vertiente del Golfo, aún hace falta aclarar su distribución, ya que la distinción con $X$. ferrugineus es reciente.

Un caso particularmente importante es con respecto a la especie $X$. palatus; esta se encuentra principalmente reportada en los estados de Colima, Jalisco, Nayarit (Burgos y Equihua, 2007), y un sólo reporte en Oaxaca (Atkinson \& Equihua, 1988) (Apéndice 2); de acuerdo con Rzedowski (1978) comprende zonas bosque tropical caducifolio y coincide con la Sierra Madre del Sur, por lo que es probable que esta especie sea exclusiva de estas regiones, donde se encuentran distribuidos los bosque secos, además coinciden con Martínez y Atkinson (1986) quienes reportan a esta especie en un bosque tropical caducifolio en Jalisco. Sin embargo es necesario realizar recolectas enfocadas en esta región para definir con certeza la distribución, que de acuerdo con Atkinson (2012) esta región es de mayor prioridad para analizar la fauna de Scolytinae presente allí.

Las especies $X$. affinis, $X$. ferrugineus y $X$. volvulus se encuentran ampliamente distribuidas para todas las áreas tropicales y subtropicales (Apéndice 2), sin embargo las primeras dos también se incluyen para zonas templadas del este de Norteamérica. Pérez-De la Cruz et al. (2009b) determinaron en el estado de Tabasco, que una precipitación entre 400 y $700 \mathrm{~mm}$ y una temperatura entre 24 y $29{ }^{\circ} \mathrm{C}$ propician el desarrollo óptimo de los escolitinos, datos que resaltan debido a que las especies más abundantes fueron $X$. volvulus y $X$. affinis. A pesar de ello Rangel et al. (2012) hacen referencia en que las poblaciones de $X$. ferrugineus y $X$. affinis pueden presentar picos poblacionales a lo largo de todo el año, aunque señalan que los factores con mayor influencia en estas dos especies son la temperatura (similar a la obtenida por Pérez-De la Cruz et al. 2009b) y la humedad, la cual es mayor al $60 \%$.

Otros estudios apoyan la amplia distribución de estas especies, la cual no solo se reconoce en México, si no en la región circumtropical, tal es el caso de Vázquez et al. (2003), quienes enlistan los escolitinos de Cuba, en donde reportan cinco especies de Xyleborus, de las cuales sólo $X$. xylographus no se encuentra en México. Otro estudio se realizó en el área central de la Amazonia (Souza et al. 2011) en donde también se registran cinco especies. En ambos casos cuatro especies coinciden ( $X$. affinis, $X$. ferrugineus, $X$. spinulosus y $X$. volvulus); sin embargo, mientras que en Cuba la especie más abundante fue $X$. volvulus, en la región amazónica fue $X$. affinis, con lo cual se confirma lo expuesto arriba, referido a su amplia distribución.

\section{CONCLUSIONES}

En México existen 18 especies del género Xyleborus, de las cuales $X$. bispinatus y $X$. vismiae son nuevos registros para México. Además se reporta por primera vez a $X$. horridus en los estados de Chiapas, Guerrero y San Luis Potosí; $X$. intrusus en Baja California Norte y Baja California Sur; $X$. spinulosus para Tamaulipas; $X$. posticus en Morelos y $X$. declivis en Chiapas y Tabasco.

El estado en donde se reporta el mayor número de especies es Veracruz (14) y los de menor son Baja California Norte, México, Colima y Querétaro con 2, 2, 1 y 1 especies respectivamente. La vertiente del Golfo de México presenta un mayor número de especies registradas, sin embargo, es preciso realizar recolectas en algunas regiones del país para definir con certeza, la distribución y los patrones biogeográficos de las especies mexicanas de Xyleborus.

AGRADECIMIENTOS. Al Dr. Saúl Sánchez Soto del Colegio de Postgraduados, Campus Tabasco, por su apoyo en la colocación de trampas. A la Biól. Rosamond Ione Coates Lutes por proporcionar las facilidades de colecta en la Estación de Biología Tropical "Los Tuxtlas”. Al M. en C. Artemio Hernández, M. en C. Lidia Guzmán, Biól. Edson Espinoza y P. de Biól. Ivette Ríos por su apoyo en las colectas.

\section{LITERATURA CITADA}

Atkinson, T. H. 2014. Bark and Ambrosia Beetles. Disponible en: http://www.barkbeetles.info/about.php. (Consultada en 2014).

Atkinson, T.H. 2012. Estado de conocimiento de la taxonomía de los escarabajos descortezadores y ambrosiales de México (Coleoptera:Curculionidae: Scolytinae). pp. 13-27. In: Memorias del XVI Simposio Nacional de Parasitología Forestal, Cuernavaca, Morelos, México.

Atkinson, T. H. \& Equihua-Martínez, A. 1986a. Biology of the Scolytidae and Platypodidae (Coleoptera) in a tropical deciduous forest at Chamela, Jalisco, Mexico. Florida Entomologist, 62: 303-310.

Atkinson T. H. \& Equihua-Martínez, A. 1986b. Biology of bark and ambrosia beetles (Coleoptera: Scolytidae and Platypodidae) of a tropical rain forest in Southeastern Mexico with an annotated checklist of species. Annals of the Entomological Society of America, 79: 414-423.

Atkinson T. H. \& Equihua-Martínez, A. 1988. Notas sobre la Biología de Scolytidae y Platypodidae (Coleoptera) de México y Centroamérica. Folia Entomológica Mexicana, 76: 83-105.

Atkinson, T. H., Martínez-Fernández, E., Saucedo-Céspedes, E. \& Burgos-Solorio, A. 1986a. Scolytidae y Platypodidae (Coleoptera) asociados a selva baja y comunidades derivadas en el estado de Morelos, México. Folia Entomológica Mexicana, 69: 41-82.

Atkinson, T. H., Saucedo-Céspedes, E., Martínez-Fernández, E. \& Burgos-Solorio, A. 1986b. Coleópteros Scolytidae y Platypodidae asociados con las comunidades vegetales de clima templado 
y frío en el estado de Morelos, México. Acta Zoológica Mexicana (n.s.), 17: 1-58.

Atkinson, T. H., Carrillo, D., Duncan, R. E. \& Peña, J. E. 2013. Occurrence of Xyleborus bispinatus (Coleoptera: Scolytinae) Eichhoff in southern Florida. Zootaxa, 3669: 96-100.

Bright, D. E. Jr. 1968. Review of the tribe Xyleborini in America North of Mexico (Coleoptera: Scolytidae). The Cannadian Entomologist, 100: 1288-1323.

Bright, D. E. 2014. A Catalog of Scolytidae and Platypodidae (Coleoptera), Supplement 3 (2000-2010), with notes on subfamily and tribal reclassifications. University of Nebraska-Lincoln. Insecta Mundi. 861. http://digitalcommons.unl.edu/insectamundi/861

Burgos-Solorio A. \& Equihua M., A. 2007. Platypodidae y Scolytidae (Coleoptera) de Jalisco, México. Dugesiana, 14: 59-82.

Cibrián, D., Méndez, J., Campos, R., Yates III, O. \& Flores, J. 1995. Insectos forestales de México. Universidad Autónoma Chapingo, estado de México, México. 450 pp.

Dirección General de Sanidad Vegetal. 2014. Escarabajo ambrosial del Laurel Rojo Xyleborus glabratus, Eichhoff (Coleoptera:Curculionidae:Scolytinae). Servicio Nacional de Sanidad, Inocuidad y Calidad Agroalimentaria. Secretaría de Agricultura, Ganadería, Desarrollo Rural, Pesca y Alimentación. México. 11 pp.

Equihua M., A. \& Burgos S., A. 2002. Scolytidae. pp. 539-557. In: Llorente. B. J. \& J. J. Morrone (Eds.). Biodiversidad, Taxonomía y Biogeografía de artrópodos de México: Hacia una síntesis de su conocimiento Vol. III. CONABIO-IBUNAM. México.

Estrada, V. A. \& Atkinson, T. H. 1988. Scolytidae y Platypodidae (Coleoptera) de Escárcega, Campeche, México. Biogeografía, biología, importancia económica y una lista comentada de especies. Anales del Instituto de Biología, Serie Zoología, 58: 199-220.

Hulcr, J., \& Cognato, A. I. 2010. New genera of Palaeotropical Xyleborini (Coleoptera: Curculionidae:Scolytinae) based on congruence between morphological and molecular characters. Zootaxa, 2717: 1-33.

Hulcr, J., Dole, S. A., Beaver, R. A. \& Cognato, A. I. 2007. Cladistic review of generic taxonomic characters in Xyleborina (Coleoptera: Curculionidae: Scolytinae). Systematic Entomology, 32: 568-584.

Lombardero, M. J. 1996. Representantes de la tribu Xyleborini LeConte, 1876 (Coleoptera:Scolytidae) en la Península Ibérica. Boletín de la Asociación Española de Entomología, 20:173-191.

Noguera-Martínez F. A. \& Atkinson, T. H. 1990. Biogeography and biology of bark and ambrosia beetles (Coleoptera: Scolytidae and Platypodidae) of a mesic montane forest in Mexico, with an annotated checklist of species. Annals of the Entomological Society of America, 83: 453-466.
Pérez-De La Cruz, M., Equihua-Martínez, A., Romero-Nápoles, J., Valdez-Carrasco, J. \& De La Cruz-Pérez, A. 2009a. Claves para la identificación de escolitinos (Coleoptera: Curculionidae: Scolitinae) asociados al agroecosistema del cacao en el sur de México. Boletín del Museo de Entomología de la Universidad del Valle, 10: 14-29.

Pérez-De La Cruz M., Equihua-Martínez, A., Romero Nápoles, J., Sánchez-Soto, S. \& García-López, E. 2009b. Diversidad, fluctuación poblacional y plantas huésped de escolitinos (Coleoptera:Curculionidae) asociados con el agroecosistema cacao en Tabasco, México. Revista Mexicana de Biodiversidad, 80: 779791.

Pulgarín D., J. A. 2012. Scolytinae (Coleoptera:Curculionidae) Asociados a dos maderas en un bosque muy húmedo premontano (Antioquia, Colombia). Revista Facultad Nacional de Agronomía Medellín, 65: 6459-6466.

Rabaglia R. J., Dole, S. A. \& Cognato, A. I. 2006. Review of American Xyleborina (Coleoptera: Curculionidae: Scolytinae) occurring North of México, with an illustrated key. Annals of the Entomological Society of America, 99: 1034-1056.

Rangel R., Pérez, M., Sánchez, S. \& Capello, S. 2012. Fluctuación poblacional de Xyleborus ferrugineus y $X$. affinis (Coleoptera: Curculionidae) en ecosistemas de Tabasco, México. Revista de Biología Tropical, 60: 1577-1588.

Romero N. J., Anaya R., S., Equihua Martínez, A. \& Mejía G., H. 1997. Lista de Scolytidae y Platypodidae de México (Insecta: Coleoptera). Acta Zoológica Mexicana (n.s.), 70: 36-53.

Rzedowski, J. 1978. Vegetación de México. Limusa. México

Salinas-Moreno Y., Vargas M., C. F., Zúniga, G., Víctor A., J. \& Hayes, J. L. 2010. Atlas de distribución geográfica de los descortezadores del género Dendroctonus (Curculionidae:Scolytidae) en México. Instituto Politécnico Nacional. CONAFOR. México 90.

Souza de Abreu, R. L., Greicilany de Araújo, R., Frasco V., B. \& Sales-Campos, C. 2012. Insects of the subfamily Scolytinae (Insecta:Coleoptera, Curculionidae) collected with pitfall and ethanol traps in primary forests of Central Amazonia. Psyche, 2012. 8 p. ID 480520

Vázquez M., L. L., Rodríguez P., M. \& Zorrilla, M. A. 2003. Lista de escolítidos (Coleoptera) de Cuba y sus plantas hospederas. Fitosanidad, 7: 17-21.

Wood, S. L. 1982. The bark and ambrosia beetles of North and Central America (Coleoptera:Scolytinae). A taxonomic monograph. Great Basin Naturalis Memoirs 6.

Wood, S. L. 1986. A reclassification of the genera of Scolytidae (Coleoptera). Great Basin Naturalis Memoirs 10.

\section{APÉNDICE 1. ESPECIES MEXICANAS DEL GÉNERO XYLEBORUS EN MÉXICO}

El presente listado comprende las colecciones en donde se encuentran depositadas las especies de Xyleborus en México, además de la distribución y las especies hospederas; únicamente para los nuevos registros se incluyen datos de colecta.

1. Xyleborus affinis Eichhoff, 1868. (CIIFIT, CNIN, ENCB-IPN). (Figura 3: Mapa 1).

Distribución: Baja California Sur, Campeche, Chiapas, Guerrero, Hidalgo, Jalisco, México, Michoacán, Morelos, Nayarit, Oaxaca, Puebla, Quintana Roo, Sinaloa, San Luis Potosí, Tabasco, Tamaulipas, Vera- cruz y Yucatán (Todos los estados en México). Hospederos: Acacia guameri, Acrocarpus fraxinifolius, Brosimum alicastrum, Bursera instabilis, Cedrela odorata, Celtis laevigata, Croton nitens, Dialium guianense, Erythrina sp., Leucaena pulverulenta, Manikara zapota, Mangifera indica, Metopium brownei, Persea sp., Rheedia sp., Spondias mombin, Sacharinum officinale, Tabebuia rosea, Theobroma cacao.

2. Xyleborus bispinatus Eichhoff, 1868 (Nuevo registro para México) (CIIFIT, CNIN). (Figura 3: Mapa 2). 
Distribución: Campeche (Campo de investigación forestal "El Tormento", 27/IX/1983, Estrada V., A.) y Veracruz (Estación Biológica Los Tuxtlas, 29/ XI/1989, Rojas, H. y Colín, J. L., trampa de intercepción; San Andrés Tuxtla, La Palma, 9/XI/1989, Colín, J. L. y Rojas, H.; Jalapa, 20/VIII/1983, A. Noguera, F.; Jalapa, 18/VII/1983, A. Noguera, F.; Xalapa, 26/VII/1983, A. Noguera, F.)

Hospederos: Quercus sp. y Switenia macrophylla.

3. Xyleborus declivis Eichhoff, 1869. (CIIFIT, CNIN). (Figura 3: Mapa 3).

Distribución: Chiapas (Nuevo registro para el estado: Palenque, 24/IV/1987, Chamberlin, W. F., trampa de luz; Reserva El Ocote, 2/X/1995, G. Ortega, E, Barrera y A. Casasola), Oaxaca, Tabasco y Veracruz.

4. Xyleborus discretus Eggers, 1933. (CIIFIT). (Figura 3: Mapa 4).

Distribución: Chiapas (Palenque) y Veracruz (Uxpanapa y Vista Hermosa).

Hospederos: Dialium guianense, Heliocarpus appendiculatus, Inga sp. y Schizolobium parahybum.

5. Xyleborus ferrugineus (Fabricius, 1801). (CIIFIT, CNIN, ENCB-IPN). (Figura 3: Mapa 5).

Distribución: Campeche, Chiapas, Distrito Federal, Guerrero, Hidalgo, Jalisco, Michoacán, Morelos, Nayarit, Nuevo León, Oaxaca, Puebla, Querétaro, Quintana Roo, San Luis Potosí, Sinaloa, Sonora, Tabasco, Tamaulipas, Veracruz y Yucatán (Todos los estados en México).

Hospederos: Acacia guameri, Acrocarpus fraxinifolius, Apidosperma megalocarpon, Artocarpus altilis, Brosimum alicastrum, Bursera sp., Bursera simaruba, Cecropia obtusifolia, Cedrela sp., Cedrela odorata, Delonix regia, Dialium guianense, Erythrina sp., Erythrina brevifolia, Ficus sp., Ficus retusa nítida, Gliricidia sepium, Guarea sp., Heliocarpus sp., Heliocarpus appendiculatus, Heliocarpus pallidus, Lysiloma bahamensis, Manikara zapota, Mangifera indica, Pinus sp., Quercus sp., Rheedia sp., Sciadodendron excelsum, Spondias mombin, Swietenia macrophylla, Tabebuia rosea, Terminalia amazonica, Theobroma cacao, Thouinidium decandrum, Urera sp. y Vitex guameri.

6. Xyleborus horridus Eichhoff, 1869. (CIIFIT). (Figura 3: Mapa 6).

Distribución: Campeche, Chiapas (Nuevo registro para el estado: El Chorreadero, 26/VI/1990, Thomas, M. C.; Parque Laguna Bélgica, 3/X/1987, Turnbow, R. H., trampa de luz de vapor de mercurio y luz ultravioleta), Guerrero (Nuevo registro para el estado: 24 Km NE jct hwy 200 on hwy 134, 19/VII/1985, Turnbow, R. H.), San Luis Potosí (Nuevo registro para el estado: Tamazunchale, 19/VI/1941, Dybas, H. S.), Tabasco, Tamaulipas y Veracruz.

Hospederos: Acacia guameri, Alchornea sp., Astronium graveolens, Brosimum alicastrum, Bursera simaruba, Hevea bresilensis, Inga sp., Lysiloma bahamensis, Piscidia communis, Trema micrantha. Zygia sp.

7. Xyleborus imbellis Blandford, 1898. (CIIFIT). (Figura 4: Mapa 7).

Distribución: Campeche, Quintana Roo, Veracruz (El Palmar y Vista Hermosa) y Tamaulipas (El Cielo). Hospederos: Hevea brasiliensis e Inga sp.

8. Xyleborus intrusus Blandford, 1898. (CIIFIT, ENCB-IPN). (Figura 4: Mapa 8).

Distribución: Baja California Norte (Nuevo registro para el estado: Las Arrastras, 7 mi N, 9/VI/1967, Sleeper, E. L.), Baja California Sur (Nuevo registro para el estado: Ramal a Los Naranjos, $24 \mathrm{~km} \mathrm{W,}$ 28/8/1994, Turnbow, R. H.; 2 mi E, 6/VII/1979, Andrews, F. G. Hardy A. R. Giulani; La Paz, $10 \mathrm{mi} \mathrm{S}$, 26/VIII/1994, Turnbow, R. H. trampa de luz vapor de mercurio+ultravioleta; Los Barriles, $3 \mathrm{Km} \mathrm{S}$, 27/ VIII/1994, Turnbow, R. H. trampa de luz vapor de mercurio+ultravioleta), Chiapas, Distrito Federal, Durango, Guerrero, Hidalgo, Jalisco, México, Michoacán, Morelos, Oaxaca, Puebla y Tlaxcala.

Hospederos: Pinus sp., Pinus leiophylla, P. montezumae, P. oocarpa, P. patula y P. pseudostrobus.

9. Xyleborus macer Blandford, 1898. (CIIFIT). (Figura 4: Mapa 9).

Distribución: Campeche (Campo de Investigación forestal "El Tormento"), Chiapas (Ozocoautla, Palenque y Villa Flores), Tabasco y Veracruz (Balzapote, Dos Amates y Lago de Catemaco).

Hospederos: Lysiloma bahamensis.

10. Xyleborus morulus Blandford, 1898. (CIIFIT). (Figura 4: Mapa 10).

Distribución: Michoacán (Patuán).

Hospederos: Spondias mombin.

11. Xyleborus palatus Wood, 1974. (CIIFIT). (Figura 4: Mapa 11).

Distribución: Colima (Armería, Cihuatlán, Colima), Jalisco (Chamela y Volcán de Colima), Nayarit (Laguna Sta. María y San Blas) y Oaxaca (Teotitlán).

Hospederos: Caesalpinia platyloba, Conocarpus erecta, Cordia dentate, Croton pseudoniveus, Lonchocarpus guatemalensis, Mangifera indica, Pithecellobium sp.

12. Xyleborus posticus Eichhoff, 1869. (CIIFIT). (Figura 4: Mapa 12).

Distribución: Campeche, Chiapas, Hidalgo, Morelos (Nuevo registro para el estado: Amatlán, Tepoztlán, 
10/XII/2013, Pérez S., M. y Espinoza G., E. M.), San Luis Potosí, Tabasco, Tamaulipas y Veracruz. Hospederos: Astronium graveolens, Cecropia obtusifolia, Cupressus lindleyi, Erythrina sp., Erythrina americana, Ficus elástica, Heliocarpus sp., Heliocarpus appendiculatus, Leucaena pulverulenta,

13. Xyleborus spathipennis Eichhoff, 1868. (CIIFIT). (Figura 5: Mapa 13).

Distribución: Oaxaca (Nicolás Bravo).

Hospederos: Pouteria sapota

14. Xyleborus spinulosus Blandford, 1898. (CIIFIT). (Figura 5: Mapa 14).

Distribución: Campeche, Chiapas, Guerrero, Jalisco, Nayarit, Oaxaca, Quintana Roo, Tabasco, Tamaulipas (Nuevo registro para el estado: Camino a Alto Cielo, 15/VIII/2009, Atkinson, T. H.) y Veracruz. Hospederos: Ficus cotiniifolia, Guettarda combsii, Lonchocarpus rugosus, Lysiloma bahamensis, Mangifera indica; Metopium brownei, Nectandra sp., Nectandra ambigens, Pouteria campechiana, Sebastiana adenophora, Trophis racemosa, Vitex guameri

15. Xyleborus squamulatus Eichhoff, 1869. (CIIFIT). (Figura 5: Mapa 15).

Distribución: Chiapas (El Bosque), Oaxaca (Valle Nacional) y Veracruz (Xalapa).

16. Xyleborus titubanter Schedl, 1948. (Figura 5: Mapa 16). Distribución: Puebla (Tezuitlán) y Veracruz (Xalapa). Hospederos: Alnus sp.
17. Xyleborus vismiae Wood, 1974. (Nuevo registro para México). (Figura 5: Mapa 16).

Distribución: Veracruz (Sontecomapan, 16 Km O, 10/IV/1993, Wappes, J. E.)

18. Xyleborus volvulus (Fabricius, 1775). (CIIFIT). (Figura 5: Mapa 17).

Distribución: Baja California Norte, Baja California Sur, Campeche, Chiapas, Distrito Federal, Guerrero, Jalisco, México, Michoacán, Morelos, Nayarit, Nuevo León, Oaxaca, Puebla, Quintana Roo, Sinaloa, San Luis Potosí, Tabasco, Tamaulipas, Veracruz y Yucatán.

Hospederos: Acacia guameri, Acacia pennatula, Acrocarpus fraxinifolius, Aspidosperma megalocarpon, Brosimum alicastrum, Bursera sp., Bursera microphylla, Bursera arbórea, Bursera fagaroides, Bursera instabilis, Bursera simaruba, Carica papaya, Casuarina equisetifolia, Cedrela odorata, Croton nitens, Cupania glabra, Delonix regia, Dialium guianense, Erythrina sp., Erythrina brevifolia, Ficus sp., Ficus cotinifolia, Ficus retusa nítida, Heliocarpus pallidus, Hurra poliandra, Leucaena pulverulenta, Mangifera indica, Metopium brownei, Nectandra ambigens, Schizolobium parahybum, Sciadodendron excelsum, Sebastiana adenophora, Spondias sp., Spondias mombin, Tapirira sp., Terminalia amazónica, Theobroma cacao, Thouinidium decandrum, Trophis racemosa, Vitex guameri. 
APÉNDICE 2. MAPAS DE DISTRIBUCIÓN DE LAS ESPECIES MEXICANAS DEL GÉNERO XYLEBORUS
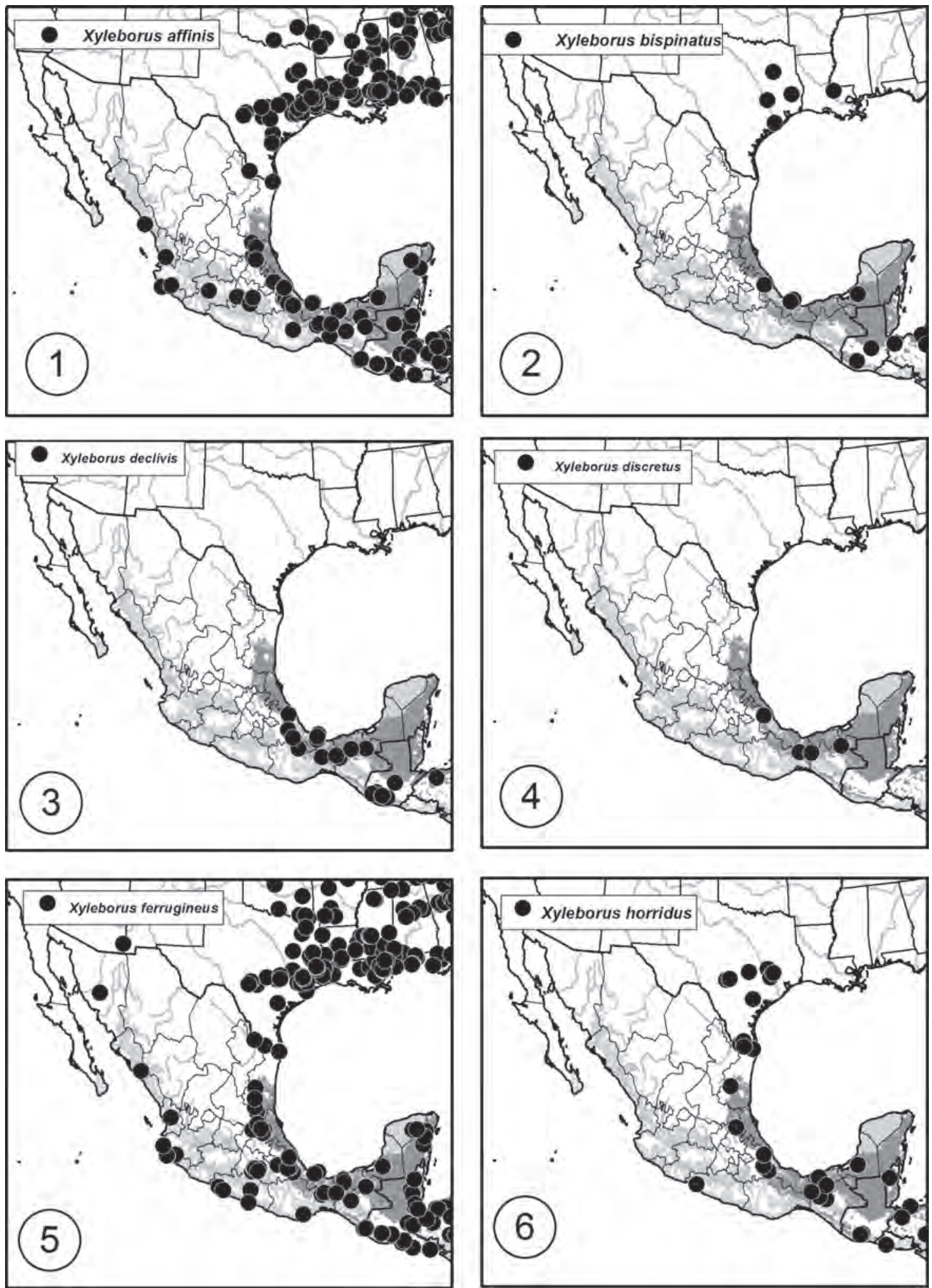

Figura 3. Mapas de distribución de las especies mexicanas de Xyleborus; 1 : $X$. affinis, 2: $X$. bispinatus, 3: $X$. declivis, 4: $X$. discretus, 5: $X$. ferrugineus y 6: X. horridus. 

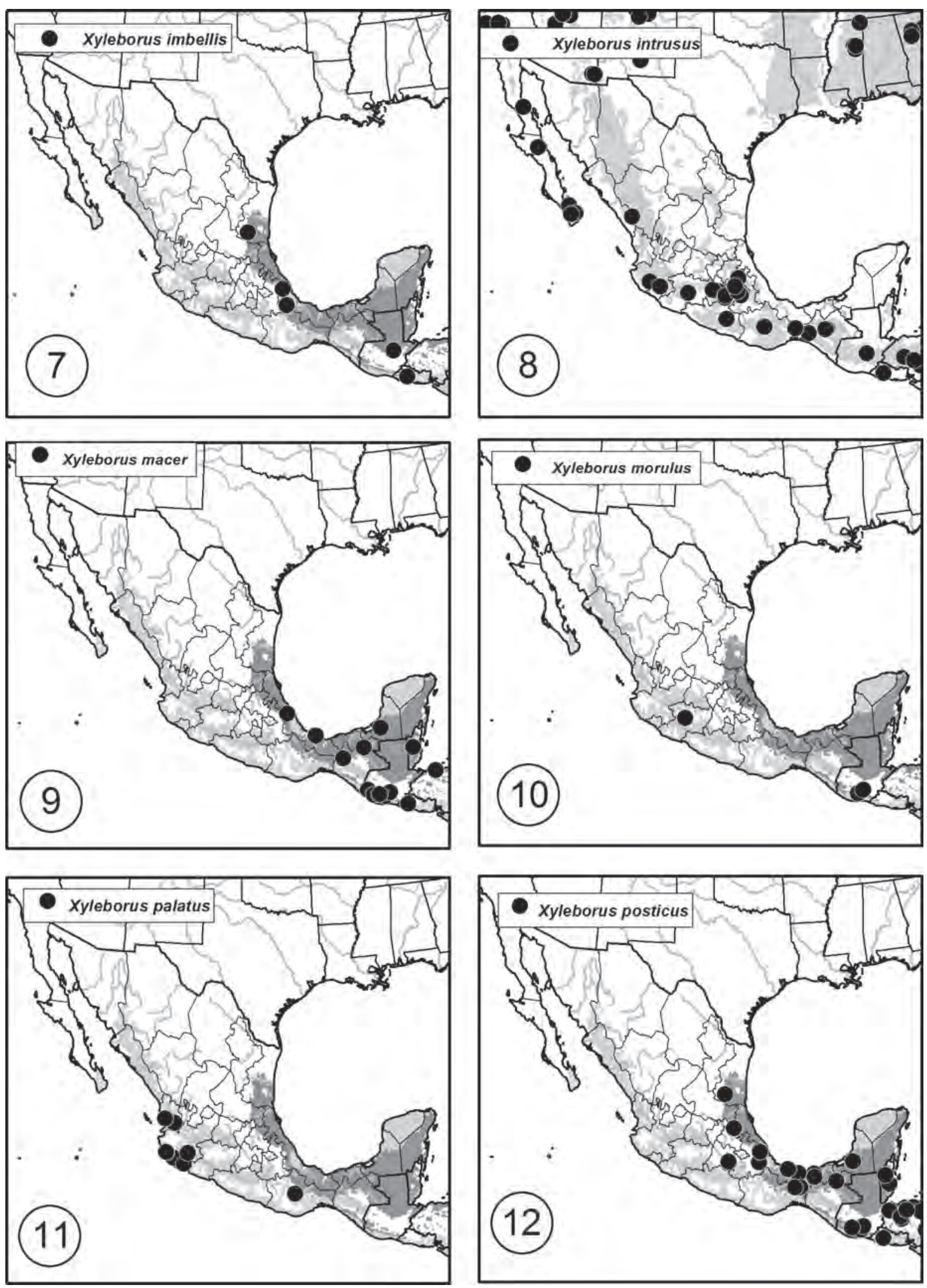

Figura 4. Mapas de distribución de las especies mexicanas de Xyleborus 7: $X$. imbellis, 8: $X$. intrusus, 9: $X$. macer, 10: $X$. morulus, 11: $X$ palatus y 12: $X$. posticus. 

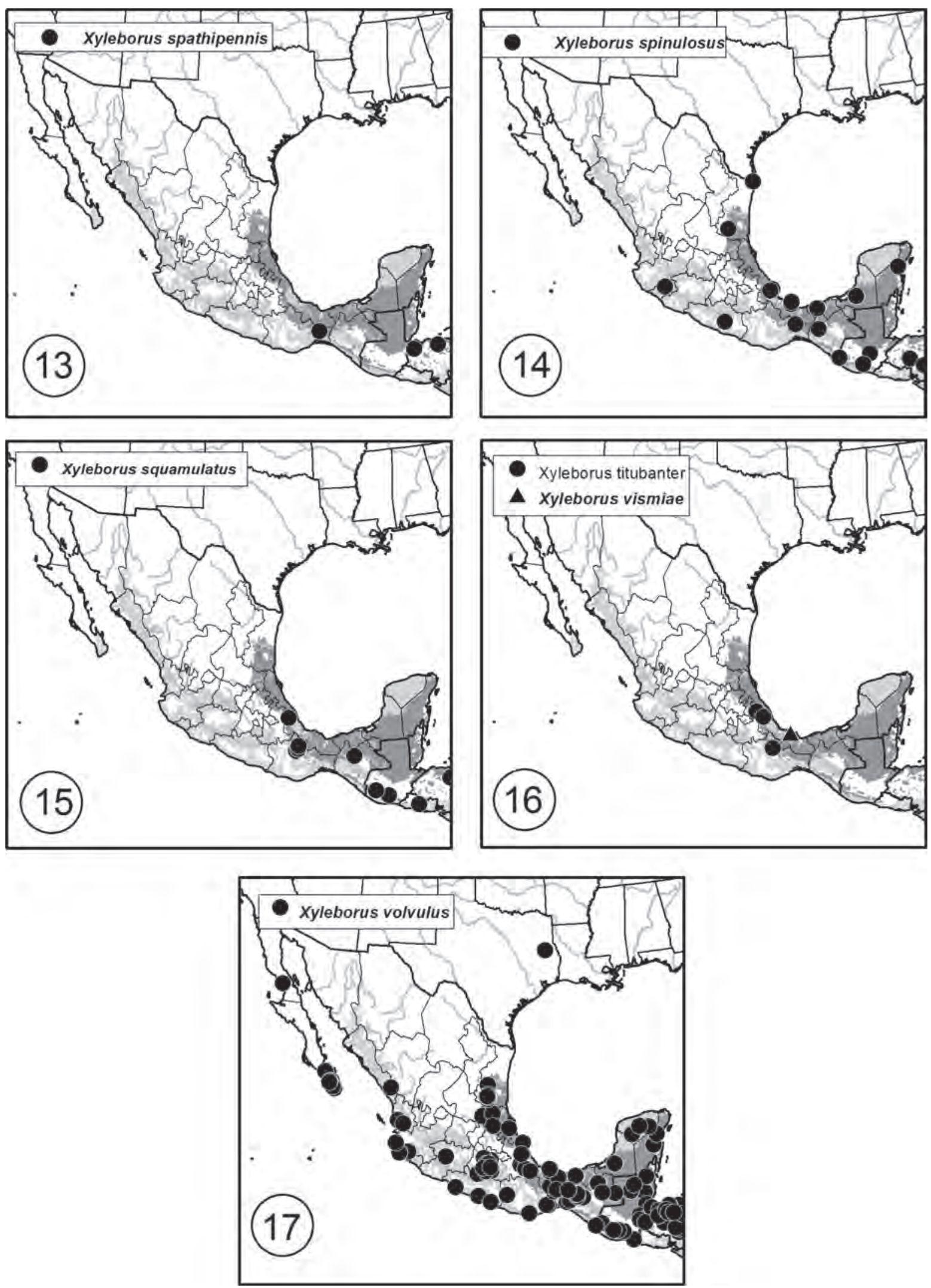

Figura 5. Mapas de distribución de las especies mexicanas de Xyleborus; 13: X. spathipennis, 14: X. spinulosus, 15: $X$. squamulatus, 16: $X$. titubanter y $X$. vismiae, y 17: $X$. volvulus. 